\title{
A Measure of Non-convexity in the Plane and the Minkowski Sum
}

\author{
R.N. Karasev
}

Received: 24 March 2009 / Revised: 7 March 2010 / Accepted: 7 March 2010 /

Published online: 24 March 2010

(C) Springer Science+Business Media, LLC 2010

\begin{abstract}
In this paper a measure of non-convexity for a simple polygonal region in the plane is introduced. It is proved that for "not far from convex" regions this measure does not decrease under the Minkowski sum operation, and guarantees that the Minkowski sum has no "holes".
\end{abstract}

Keywords Minkowski sum · Weak convexity

\section{Introduction}

Let us state the definition of the Minkowski sum of two sets $A, B \subset \mathbb{R}^{d}$.

Definition 1 The Minkowski sum is

$$
A+B=\{a+b: a \in A, b \in B\}
$$

In this paper we consider Minkowski sums in the plane. It is well-known that the Minkowski sum of two convex sets is again convex. In the case of convex polygons it is computed by a simple "edge merging and slope sorting" algorithm. If we consider non-convex polygons, the computation of the Minkowski sum may require more complicated algorithms, see [1-3] for example. In the cited papers the problem of finding the Minkowski sum arose from packing or motion planning problems. Indeed, the set of possible shifts of a region $A$ that intersect another region $B$ is the Minkowski sum $(-A)+B$, where minus denotes the reflection w.r.t. the origin.

This research was partially supported by the Dynasty Foundation.

R.N. Karasev $(\bowtie)$

Dept. of Mathematics, Moscow Institute of Physics and Technology, Institutskiy per. 9,

Dolgoprudny 141700, Russia

e-mail: r_n_karasev@mail.ru 
The most straightforward way to find the Minkowski sum of non-convex regions is to partition every non-convex region into convex polygons, calculate Minkowski sums of parts, and then take the union [1]. In some practical applications, where the regions are essentially non-convex, this approach can be too complicated, in such cases it is convenient to use the intuitive "orbital" (or "sliding") methods, see [2,3]. The latter methods deal with non-convex regions quite well, but the essential (and hard) part of these methods is finding "holes" in the Minkowski sum. Therefore, it is important to give a computable criterion for the Minkowski sum to have no "holes".

In Sect. 2 we define a measure of non-convexity aco $K$ for a simply-connected polygonal region (simple polygon) $K$ in the plane so that aco $K \leq 0$ in general and aco $K=0$ iff $K$ is convex. This measure of non-convexity uses some essential properties of $\mathbb{R}^{2}$, some other definitions of non-convexity measures valid for spaces of arbitrary (even infinite) dimensions are reviewed in [4]. Another general non-convexity measure based on the path metric in $K$ can be found in [5].

The main result is stated as follows.

Theorem 1 Suppose $K$ and $L$ are simple polygons, and aco $K$, aco $L>-\pi$. Then $K+L$ is a simple polygon and

$$
\operatorname{aco}(K+L) \geq \min \{\operatorname{aco} K, \operatorname{aco} L\} .
$$

This theorem shows that the property aco $K>-\pi$ is stable under the Minkowski sum, and in this case the sum of an arbitrary number of simple polygons is simple.

In the proof of Theorem 1 we use the following fact, which has its own value. It generalizes the separation theorem for convex sets.

Theorem 2 Suppose $K$ is a simple polygon with aco $K>-\pi$. Then for any point $x \notin K$ there exist an angular region $A$ with apex $x$, such that $\angle A=\pi+\operatorname{aco} K$ and

$$
A \cap K=\emptyset .
$$

\section{Definition of Angular Convexity}

Now we make some definitions and fix some notation.

Definition 2 The sequence of points $v_{1}, \ldots, v_{n} \in \mathbb{R}^{2}$ (vertices) and the corresponding segments $v_{1} v_{2}, \ldots, v_{n-1} v_{n}$ (edges) is called a polyline. We require that the consecutive vertices do not coincide, $v_{i} \neq v_{i+1}$.

For a polyline $P=v_{1} \cdots v_{n}$ we call the sequence of vectors $v_{2}-v_{1}, \ldots, v_{n}-v_{n-1}$ the shift sequence and denote it $S(P)$.

Definition 3 We call a polyline $v_{1} v_{2} \cdots v_{n+1}$ closed if $v_{1}=v_{n+1}$. In this case we often index its vertices modulo $n$.

Definition 4 We call a polyline simple if it has no self-intersections, i.e. its edges may intersect in one point if they are consecutive, otherwise they do not intersect. In a closed polyline we, of course, allow the first vertex to equal the last one. 
Definition 5 We call a compact set $K \subset \mathbb{R}^{2}$ a simple polygon if its boundary is a closed simple polyline.

It is obvious that generally the Minkowski sum of two simple polygons can be not simple, speaking informally it can have "holes". The objective of this paper is to find some sufficient conditions on the polygons that guarantee that the Minkowski sum is simple. We need some more definitions.

Definition 6 For two plane vectors define the skew product

$$
[v, w]=v_{x} w_{y}-v_{y} w_{x}
$$

Two non-zero vectors $v, w$ are called parallel if $v=\alpha w$. In this case, if $\alpha<0$ we call the vectors opposite.

Definition 7 For two non-opposite vectors $v, w$ denote $\angle(v, w)$ the angle between vectors with sign, positive if $[v, w]>0$, negative if $[v, w]<0$.

Definition 8 If the shift sequence of a polyline $P$ does not contain a consecutive pair of opposite vectors, we call $P$ non-reverse.

Definition 9 Let a polyline $P$ with shift sequence $S(P)=\left(s_{1}, \ldots, s_{n}\right)$ be nonreverse. The rotation of $P$ (the same as rotation of $S(P)$ ) is

$$
\operatorname{rot} P=\sum_{i=1}^{n-1} \angle\left(s_{i}, s_{i+1}\right) \text {. }
$$

If the polyline has 2 vertices we put rot $P=0$.

If the polyline $P$ is closed, then (the indices are modulo $n$ )

$$
\operatorname{rot} P=\sum_{i=1}^{n} \angle\left(s_{i}, s_{i+1}\right) .
$$

Definition 10 Let $P$ be a non-reverse polyline. Denote the angular convexity of $P$

$$
\operatorname{aco} P=\min _{L \subseteq P} \operatorname{rot} L
$$

where the minimum is taken over subpolylines $L \subseteq P$ (obtained from $P$ by removing some vertices from its front and/or its back).

Definition 11 Let $K$ be a simple polygon and $P=\partial K$ be oriented so that $\operatorname{rot} P=2 \pi$. Denote the angular convexity of $K$

$$
\operatorname{aco} K=\min _{L \subseteq P} \operatorname{rot} L
$$

where the minimum is taken over simple polylines $L \subseteq P$, oriented along $P$. 
Fig. 1 Slope diagram and the angular convexity of a polygon, $T$ is the full length

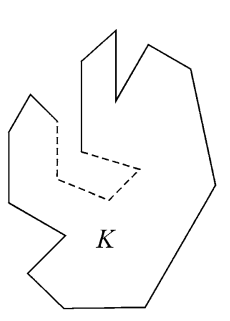

$\uparrow$

।

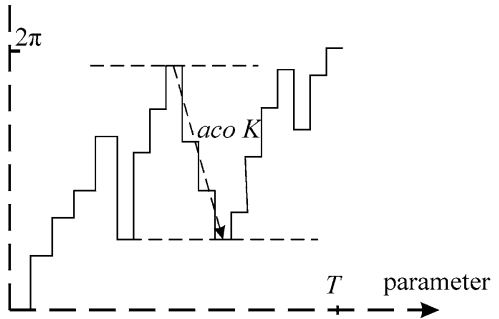

The angular convexity of a polygon $K$ is illustrated on its slope diagram (dependance of the slope angle on the anticlockwise parameter) in Fig. 1.

\section{The Sorted Sum of Polylines}

Here we formulate and prove some lemmas that generalize the algorithm of finding Minkowski sum for convex polygons by edge sorting. We start from the definition.

Definition 12 Denote the concatenation of sequences $S$ and $S^{\prime}$ by $S \circ S^{\prime}$. Denote the first element of non-empty $S$ as head $S$, denote the sequence $S$ without its first element by tail $S$.

Definition 13 Suppose two polylines $P=v_{1}, \ldots, v_{n+1}$ and $Q=w_{1}, \ldots, w_{m+1}$ are non-reverse, let their shift sequences be $S(P)=s_{1}, \ldots, s_{n}$ and $S(Q)=t_{1}, \ldots, t_{m}$. Suppose also that $\angle\left(s_{1}, t_{1}\right)=0$ and $\operatorname{rot} P=\operatorname{rot} Q$.

Define the sorted sum $R=P+{ }_{s} Q$ as follows. Let the first vertex be $v_{1}+w_{1}$, let the shift sequence $S\left(P+{ }_{s} Q\right)$ be formed by the following rule:

(1) Start from $S_{1}=S(P), S_{2}=S(Q), S_{3}=\emptyset$

(2) If $S_{1}=\emptyset$ and $S_{2}=\emptyset$ then put $S\left(P+{ }_{s} Q\right)=S_{3}$ and quit

(3) If $S_{1}=\emptyset$ then put $S\left(P+{ }_{s} Q\right)=S_{3} \circ S_{2}$ and quit

(4) If $S_{2}=\emptyset$ then put $S\left(P+{ }_{s} Q\right)=S_{3} \circ S_{1}$ and quit

(5) If $\operatorname{rot} S_{1} \geq \operatorname{rot} S_{2}$, then put $S_{3}=S_{3}$ o head $S_{1}, S_{1}=$ tail $S_{1}$ and go to step 2

(6) If $\operatorname{rot} S_{1}<\operatorname{rot} S_{2}$, then put $S_{3}=S_{3} \circ$ head $S_{2}, S_{2}=$ tail $S_{2}$ and go to step 2.

Informally, we merge the shifts from two sequences in such a way that from the two possibilities to choose the next shift we always choose the "rightmost" one, preferring the first sequence if the directions coincide. The condition that the starts and the ends of the shift sequences have the same direction, and the rotations are the same, can be relaxed in general, but it is crucial in Lemmas 2, 3, and 4. An example of a sorted sum is shown in Fig. 2.

First, we show how the sorted sum is related to the Minkowski sum. 
Fig. 2 The Minkowski sum and the sorted sum of two polylines
Curve 1

Curve 2

Sorted sum and

Minkowski sum

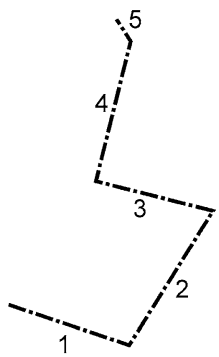

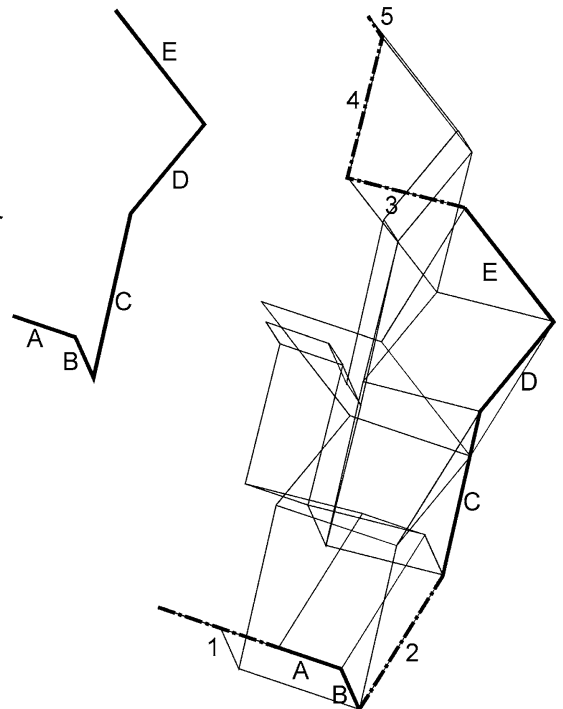

Lemma 1 Let the polylines $P$ and $Q$ be as in Definition 13 and let them have piecewise linear parameterizations

$$
P=\{p(t): t \in[0,1]\}, \quad Q=\{q(t): t \in[0,1]\}
$$

Then there are non-decreasing piece-wise linear functions (onto) $\phi, \psi:[0,1] \rightarrow$ $[0,1]$ such that

$$
r(t)=p(\phi(t))+q(\psi(t))
$$

is a parameterization of $R$, in particular $P+{ }_{s} Q \subseteq P+Q$ (the Minkowski sum of $P$ and $Q$ as subsets of the plane).

Proof Let $|S(P)|=n,|S(Q)|=m$, then $\left|S\left(P+{ }_{s} Q\right)\right|=n+m$. Without loss of generality we may assume that the parameterization $p$ maps points $0, \frac{1}{n}, \frac{2}{n}, \ldots, \frac{n-1}{n}, 1$ to the vertices of $P$, and $q$ maps points $0, \frac{1}{m}, \frac{2}{m}, \ldots, \frac{m-1}{m}, 1$ to the vertices of $Q$.

The functions $\phi$ and $\psi$ will be continuous, piece-wise linear. We will construct them so that $\phi$ on a segment $\left[\frac{i-1}{n+m}, \frac{i}{n+m}\right](i=1, \ldots, n+m)$ is either constant or linearly increases by $\frac{1}{n}$, and $\psi$ on the same segment is either constant or linearly increases by $\frac{1}{m}$.

By definition put: if the $i$-th shift in $S\left(P+{ }_{s} Q\right)$ is taken from $S(P)$, then $\phi$ increases and $\psi$ remains constant, if it is taken from $S(Q), \phi$ remains constant and $\psi$ increases.

In the first case denote $S\left(P+{ }_{s} Q\right)=S^{\prime} \circ s_{i} \circ S^{\prime \prime}$, where $\left|S^{\prime}\right|=i-1$, then for $t_{1}=\frac{i-1}{n+m}$ and $t_{2}=\frac{i}{n+m}$ 


$$
\begin{aligned}
p\left(\phi\left(t_{1}\right)\right)+q\left(\psi\left(t_{1}\right)\right) & =v_{1}+w_{1}+\sum S^{\prime} \cap S(P)+\sum S^{\prime} \cap S(Q) \\
& =v_{1}+w_{1}+\sum S^{\prime} \in P+{ }_{s} Q,
\end{aligned}
$$

and

$$
\begin{aligned}
p\left(\phi\left(t_{2}\right)\right)+q\left(\psi\left(t_{2}\right)\right) & =v_{1}+w_{1}+\sum S^{\prime} \cap S(P)+s_{k}+\sum S^{\prime} \cap S(Q) \\
& =v_{1}+w_{1}+\sum S^{\prime}+s_{i} \in P+{ }_{s} Q
\end{aligned}
$$

The above equations mean that the points $p\left(\phi\left(t_{1}\right)\right)+q\left(\psi\left(t_{1}\right)\right)$ and $p\left(\phi\left(t_{2}\right)\right)+$ $q\left(\psi\left(t_{2}\right)\right)$ are two consecutive vertices of the polyline $P+{ }_{s} Q$, and for $t \in\left[t_{1}, t_{2}\right]$ the point $p(\phi(t))+q(\psi(t))$ is on the corresponding segment of $P+{ }_{s} Q$.

The second case is considered similarly.

Lemma 2 Suppose $P, Q, R$ are as in Definition 13. If aco $P$, aco $Q>-\pi$, then $R=$ $P+{ }_{s} Q$ is non-reverse.

Proof Assume the contrary: its consecutive shifts $s_{k}$ and $t_{l}$ (indexed as they were in $P$ and $Q$ ) are opposite. Take the sequences $S_{1}$ and $S_{2}$ from the definition in the state before adding $s_{k}$ and $t_{l}$ to $S_{3}$, so $s_{k}=$ head $S_{1}, t_{l}=$ head $S_{2}$.

Since $s_{k}$ and $t_{l}$ are opposite $\operatorname{rot} S_{1}=\operatorname{rot} S_{2}+(2 N+1) \pi$, for some $N \in \mathbb{Z}$. Since $s_{k}$ comes before $t_{l}$ in $S\left(P+{ }_{s} Q\right)$, then $\operatorname{rot} S_{1} \geq \operatorname{rot} S_{2}$. Thus

$$
\operatorname{rot} S_{1} \geq \operatorname{rot} S_{2}+\pi
$$

If $\left|S_{1}\right| \geq 2$, then by the construction $\operatorname{rot} \operatorname{tail} S_{1} \leq \operatorname{rot} S_{2}$, so $\angle$ (head $S_{1}$, head tail $\left.S_{1}\right) \geq \pi$, which cannot be the case. If $\left|S_{1}\right|=1$, then $\operatorname{rot} S_{2} \leq-\pi$, which contradicts with the angular convexity condition.

Lemma 3 Suppose $P, Q, R$ are as in Definition 13. If aco $P$, aco $Q>-\pi$, then $\operatorname{rot} R=\operatorname{rot} P=\operatorname{rot} Q$.

Proof Denote $\operatorname{rot} P=\operatorname{rot} Q=\alpha$. To prove $\operatorname{rot} S\left(P+{ }_{s} Q\right)=\alpha$, it is sufficient to prove the following statement: if on some cycle of the construction the last element of $S_{3}$ is from $S(P)$, then $\operatorname{rot}\left(S_{3} \circ S_{1}\right)=\alpha$, if the last element of $S_{3}$ is from $S(Q)$, then $\operatorname{rot}\left(S_{3} \circ S_{2}\right)=\alpha$.

Let us prove it by induction. If $\left|S_{3}\right| \leq 2$ the statement is true. Let $S_{3}=S_{3}^{\prime} \circ x \circ y$, if $x$ and $y$ are shifts from $S(P)$, then

$$
\operatorname{rot}\left(S_{3} \circ S_{1}\right)=\operatorname{rot}\left(S_{3}^{\prime} \circ x \circ y \circ S_{1}\right)=\alpha
$$

by the inductive assumption. The same is true if $x, y \in S(Q)$. It is left to consider the case $x \in S(P), y \in S(Q)$. Consider two cases depending on whether $S_{1}$ is empty or not.

Case 1: $S_{1}$ is non-empty. By the construction of the sorted sum we have

$$
\operatorname{rot}\left(x \circ S_{1}\right) \geq \operatorname{rot}\left(y \circ S_{2}\right) \geq \operatorname{rot} S_{1},
$$


and since $\operatorname{rot}\left(x \circ S_{1}\right)=\angle\left(x\right.$, head $\left.S_{1}\right)+\operatorname{rot} S_{1}$, then we conclude that

$$
\left|\operatorname{rot}\left(x \circ S_{1}\right)-\operatorname{rot}\left(y \circ S_{2}\right)\right|<\pi \text {. }
$$

By the inductive assumption $\operatorname{rot}\left(S_{3}^{\prime} \circ x \circ S_{1}\right)=\alpha$. The difference

$$
\begin{aligned}
\operatorname{rot}\left(S_{3} \circ S_{2}\right)-\operatorname{rot}\left(S_{3}^{\prime} \circ x \circ S_{1}\right) & =\operatorname{rot}\left(x \circ y \circ S_{2}\right)-\operatorname{rot}\left(x \circ S_{1}\right) \\
& =\angle(x, y)+\operatorname{rot}\left(y \circ S_{2}\right)-\operatorname{rot}\left(x \circ S_{1}\right)
\end{aligned}
$$

should be a multiple of $2 \pi$. But its absolute value is less than $2 \pi$, since $|\angle(x, y)|<\pi$ and $\left|\operatorname{rot}\left(x \circ S_{1}\right)-\operatorname{rot}\left(y \circ S_{2}\right)\right|<\pi$. Hence $\operatorname{rot}\left(S_{3} \circ S_{2}\right)=\alpha$.

Case 2: $S_{1}$ is empty. Then, similar to the previous case:

$$
0 \geq \operatorname{rot}\left(y \circ S_{2}\right)
$$

and the difference

$$
\operatorname{rot}\left(S_{3}^{\prime} \circ x \circ y \circ S_{2}\right)-\operatorname{rot}\left(S_{3}^{\prime} \circ x\right)=\operatorname{rot}\left(x \circ y \circ S_{2}\right)=\angle(x, y)+\operatorname{rot}\left(y \circ S_{2}\right)
$$

should be a multiple of $2 \pi$. Since $|\angle(x, y)|<\pi$ and $0 \geq \operatorname{rot}\left(y \circ S_{2}\right) \geq$ aco $Q$, this difference should be zero.

Lemma 4 Suppose $P, Q, R$ are as in Definition 13. If aco $P$, aco $Q>-\pi$, then aco $R \geq \min \{$ aco $P$, aco $Q\}$.

Proof Denote $\alpha=\operatorname{rot} P=\operatorname{rot} Q=\operatorname{rot} R, \gamma=\min \{$ aco $P$, aco $Q\}$.

Assume the contrary: for some subsequence $S$ of the sequence $S\left(P+{ }_{s} Q\right)$ we have $\operatorname{rot} S<\gamma$. It is equivalent to the following statement: the sequence $S_{3}$ in the construction of sorted sum takes two values $S_{3}^{\prime}$ and $S_{3}^{\prime \prime}$ (in this order) so that

$$
\operatorname{rot} S_{3}^{\prime \prime}<\operatorname{rot} S_{3}^{\prime}+\gamma
$$

Put $S_{3}^{\prime}=T^{\prime} \circ x, S_{3}^{\prime \prime}=T^{\prime \prime} \circ y$, and denote the corresponding values of $S_{1}$ and $S_{2}$ by $S_{1}^{\prime}, S_{2}^{\prime}, S_{1}^{\prime \prime}, S_{2}^{\prime \prime}$.

If $x$ and $y$ are from $S(P)$ then (see the proof of Lemma 3)

$$
\operatorname{rot}\left(x \circ S_{1}^{\prime}\right)=\alpha-\operatorname{rot} S_{3}^{\prime}, \quad \operatorname{rot}\left(y \circ S_{1}^{\prime \prime}\right)=\alpha-\operatorname{rot} S_{3}^{\prime \prime}
$$

and by the assumption

$$
\operatorname{rot}\left(x \circ S_{1}^{\prime}\right)<\operatorname{rot}\left(y \circ S_{1}^{\prime \prime}\right)+\gamma
$$

If $S_{1}^{\prime}=\Sigma \circ y \circ S_{1}^{\prime \prime}$ then

$$
\operatorname{rot}(x \circ \Sigma \circ y)+\operatorname{rot}\left(y \circ S_{1}^{\prime \prime}\right)<\operatorname{rot}\left(y \circ S_{1}^{\prime \prime}\right)+\gamma,
$$

and therefore, $\operatorname{rot}(x \circ \Sigma \circ y)<\gamma$, which is a contradiction with aco $P \geq \gamma$. If $x$ and $y$ are both from $S(Q)$, the same contradiction is obtained. 
Now assume that $x$ is from $S(Q), y$ is from $S(P)$, then

$$
\operatorname{rot}\left(x \circ S_{2}^{\prime}\right)=\alpha-\operatorname{rot} S_{3}^{\prime}, \quad \operatorname{rot}\left(y \circ S_{1}^{\prime \prime}\right)=\alpha-\operatorname{rot} S_{3}^{\prime \prime}
$$

and by the assumption

$$
\operatorname{rot}\left(x \circ S_{2}^{\prime}\right)<\operatorname{rot}\left(y \circ S_{1}^{\prime \prime}\right)+\gamma .
$$

By the construction $\operatorname{rot} S_{1}^{\prime} \leq \operatorname{rot}\left(x \circ S_{2}^{\prime}\right)$ (when $x$ was added to $\left.S_{3}\right)$, and

$$
\operatorname{rot} S_{1}^{\prime}<\operatorname{rot}\left(y \circ S_{1}^{\prime \prime}\right)+\gamma \text {. }
$$

If $S_{1}^{\prime}=\Sigma \circ y \circ S_{1}^{\prime \prime}$ then

$$
\operatorname{rot}(\Sigma \circ y)+\operatorname{rot}\left(y \circ S_{1}^{\prime \prime}\right)<\operatorname{rot}\left(y \circ S_{1}^{\prime \prime}\right)+\gamma,
$$

and therefore, $\operatorname{rot}(\Sigma \circ y)<\gamma$, which is a contradiction with aco $P \geq \gamma$.

The case $x$ is from $S(P), y$ is from $S(Q)$ is considered the same way.

\section{Elimination of Self-intersections}

In this section we consider a polyline $P$ that has self-intersection. Then we convert it to a polyline without self-intersections and try to describe how the rotation of $P$ changes. First we need a definition.

Definition 14 A polyline $P$ is said to be in general position, if

(1) all its vertices are distinct

(2) any two edges intersect in at most one point, the common point being either the common vertex, or in the relative interiors of both edges

(3) any three edges do not have a common point.

It is clear that, by arbitrarily small movements of the vertices, a polyline can be put to a general position.

Definition 15 Let a polyline $P$ be in general position. Suppose it is given by a piece-wise linear parameterization $P=\{p(t): t \in[a, b]\}$ and has a self-intersection $p\left(t_{1}\right)=p\left(t_{2}\right)$ for some $t_{1}<t_{2}$. Call the loop removal the transform that replaces $P$ by the concatenation of polylines $P^{\prime}=\left\{p(t): t \in\left[a, t_{1}\right]\right\}$ and $P^{\prime \prime}=\left\{p(t): t \in\left[t_{2}, b\right]\right\}$.

It is clear that generally rot $P$ changes by a multiple of $2 \pi$ after the loop removal. The following lemma tells more.

Lemma 5 Let $P$ be a polyline in general position. Suppose that the subpolyline of $P$ between $p\left(t_{1}\right)$ and $p\left(t_{2}\right)$ has rotation $>-\pi$. Then the rotation $\operatorname{rot} P$ cannot increase after loop removal. 
Fig. 3 Removing loops in a polyline

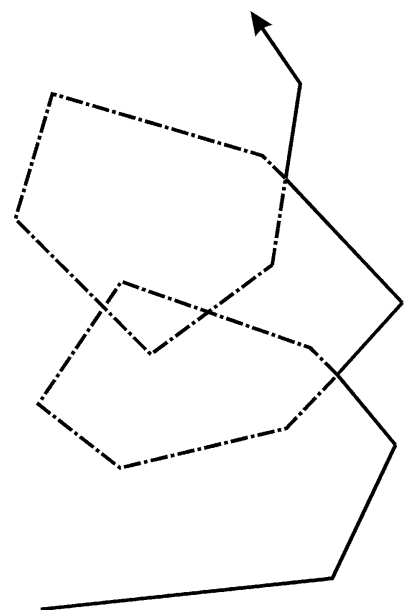

Proof Let $p\left(t_{1}\right)$ lie on the edge with direction $s_{1}, p\left(t_{2}\right)$ line on another edge with direction $s_{2}$. Denote the subpolyline between $p\left(t_{1}\right)$ and $p\left(t_{2}\right)$ by $L$.

If we identify $p\left(t_{1}\right)$ and $p\left(t_{2}\right)$ in $L$ we obtain a closed polyline, so

$$
\operatorname{rot} L+\angle\left(s_{2}, s_{1}\right)=2 \pi N, \quad N \in \mathbb{Z} .
$$

Since $\operatorname{rot} L>-\pi$ and $\left|\angle\left(s_{2}, s_{1}\right)\right|<\pi$, we obtain $\operatorname{rot} L+\angle\left(s_{2}, s_{1}\right) \geq 0$.

After removal of $L$ the rotation of the new polyline differs from $\operatorname{rot} P$ by $\angle\left(s_{1}, s_{2}\right)-\operatorname{rot} L=-\angle\left(s_{2}, s_{1}\right)-\operatorname{rot} L \leq 0$, so it does not increase.

Lemma 6 Let $P$ be a polyline in general position with aco $P>-\pi$. Then we can remove all loops in $P$ so that its rotation does not increase.

Proof Consider all the self-intersections in $P$, choose the first (w.r.t. the parameterization) such point $p\left(t_{1}\right)=p\left(t_{2}\right)$, and remove the loop between $p\left(t_{1}\right)$ and $p\left(t_{2}\right)$. Then continue such steps until all the self-intersections are removed. It is clear that the removed segments of parameterization do not intersect, each removed subpolyline had angular convexity $>-\pi$ and by Lemma 5 the angular convexity never increased.

An example of removing all loops is shown in Fig. 3.

\section{Proof of Theorem 2}

Let us give some definitions.

Definition 16 A convex hull of two rays $r_{1}$ and $r_{2}$ with common apex is called an angular region. For an angular region $A$ we denote its angular measure $\angle A=$ $\left|\angle\left(r_{1}, r_{2}\right)\right|$.

We generalize the notion of rotation to piece-wise smooth curves. 
Definition 17 Let $C=\{c(t): t \in[a, b]\}$ be a smooth curve with non-zero derivative. Then the unit tangent

$$
\tau(t)=\frac{c^{\prime}(t)}{\left|c^{\prime}(t)\right|}
$$

is well defined and can be considered as a continuous map $\tau:[a, b] \rightarrow S^{1}$ to the unit circle. Consider the universal cover $\kappa: \mathbb{R} \rightarrow S^{1}$ given by the anti-clockwise angle. The map $\tau$ is lifted continuously to a map $\tilde{\tau}:[a, b] \rightarrow \mathbb{R}$ so that $\tau=\kappa \circ \tilde{\tau}$.

Then the rotation number of $C$ is $\tilde{\tau}(b)-\tilde{\tau}(a)$.

Definition 18 Let $C=\{c(t): t \in[a, b]\}$ be a piece-wise smooth curve, composed as a concatenation of smooth curves $C_{1} \circ \cdots \circ C_{n}$. Denote the values of the parameter, where $C_{i}$ is changed to $C_{i+1}$ by $t_{i}$. We require the vectors $c_{-}^{\prime}\left(t_{i}\right)$ and $c_{+}^{\prime}\left(t_{i}\right)$ (left and right derivative) to be non-opposite.

Then the rotation number of $C$ is

$$
\operatorname{rot} C=\sum_{i=1}^{n} \operatorname{rot} C_{i}+\sum_{i=1}^{n-1} \angle\left(c_{-}^{\prime}\left(t_{i}\right), c_{+}^{\prime}\left(t_{i}\right)\right) .
$$

It is clear that if the curve $C$ is approximated by a polyline $P$ with small enough step, then $\operatorname{rot} C=\operatorname{rot} P$. The angular convexity of a curve is defined similarly.

Now we are going to prove Theorem 2. The polyline $P=\partial K$ can be approximated by a smooth curve as follows: let $v_{i}$ be its vertex with non-zero angle $\angle\left(v_{i}-v_{i-1}, v_{i+1}-v_{i}\right)$, in a small neighborhood of $v_{i}$ we replace the union of two small equal segments of $P$ (the first segment is from $v_{i}$ back along $\partial P$, the second is from $v_{i}$ forth along $\partial P$ ) by a circular arc $A_{i}$, so that the tangent becomes continuous along the resulting curves. If the segments are taken small enough, then the new boundary $P^{\prime}$ has no self-intersections. If we denote by $K^{\prime}$ the region, bounded by $P^{\prime}$, then the (tested for the separation property) point $x$ still lies outside $P^{\prime}$ for close enough approximation.

Note that $\operatorname{rot} A_{i}=\angle\left(v_{i}-v_{i-1}, v_{i+1}-v_{i}\right)$ by the construction, so it is clear that aco $P^{\prime}=\operatorname{aco} P=\gamma$.

For any two points $p_{1}, p_{2} \in P^{\prime}$ let us denote $\left[p_{1}, p_{2}\right]_{P^{\prime}}$ the subcurve of $P^{\prime}$, oriented along $P^{\prime}$ that starts at $p_{1}$ and ends at $p_{2}$. Consider the following functions of a point $p \in P^{\prime}$

$$
\gamma_{+}(p)=\min _{q \in P^{\prime}} \operatorname{rot}[p, q]_{P^{\prime}}, \quad \gamma_{-}(p)=\min _{q \in P^{\prime}} \operatorname{rot}[q, p]_{P^{\prime}}
$$

It is clear that they are continuous and $\gamma_{-}(p)+\gamma_{+}(p) \geq \gamma$.

Take the rays $r_{+}(p)$ and $r_{-}(p)$ with apex $p$ so that

$$
\angle\left(\tau(p), r_{+}(p)\right)=\gamma_{+}(p), \quad \angle\left(r_{-}(p), \tau(p)\right)=\gamma_{-}(p) .
$$

Note that the rays $r_{+}(p)$ and $r_{-}(p)$ point outside or tangentially to $K^{\prime}$, and the angle between them is at least $\pi+\gamma>0$. Denote the angular region $A(p)=\operatorname{conv}\left(r_{+}(p) \cup\right.$ $\left.r_{-}(p)\right)$. 
Fig. 4 The polygon $K^{\prime}$ and the angular region $A(p)$

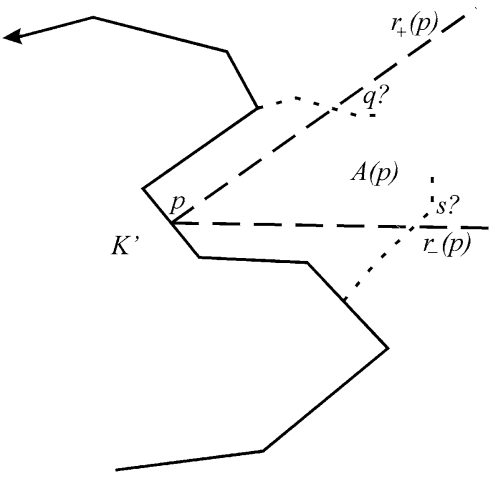

We are going to show that $K^{\prime} \cap$ int $A(p)=\emptyset$. Assume the contrary, in this case $P^{\prime}$ has to pass through the interior of $A(p)$. The situation is outlined in Fig. 4.

Let us move the point $q$ from $p$ along $P^{\prime}$. Note the first time $q$ gets inside int $A(p)$. Suppose $q$ intersects the ray $r_{+}(p)$, then the rotation of the closed (piece-wise smooth) non-self-intersecting curve $[p, q]_{P^{\prime}} \circ[q, p]$ (the last segment is a straight line segment) is $-2 \pi$, and it is clear that $\operatorname{rot}[p, q]_{P^{\prime}}$ should be less than $\gamma_{+}(p)$, which is a contradiction.

The only possibility left is that $q$ (when moving from $p$ along $P^{\prime}$ ) gets into $A(p)$ across $r_{-}(p)$. Let $q$ be the first such point on $r_{-}(p)$, in this case we consider the closed non-self-intersecting curve $[p, q]_{P^{\prime}} \circ[q, p]$ that bounds a region $L$. If we move a point $s$ backwards from $p$ along $P^{\prime}$, then it should go out of $L$, in fact it has to intersect $[q, p]$ and get into int $A(p)$. Let $s$ be the first such point on $[q, p]$, in this case the closed simple curve $[s, p]_{P^{\prime}} \circ[p, s]$ has rotation $-2 \pi$, and $\operatorname{rot}[s, p]_{P^{\prime}}<\gamma_{-}(p)$, which is a contradiction again.

Thus the theorem is proved for points $x$, close enough to $P^{\prime}$, now we prove it for any $x$ outside $P^{\prime}$. For $p \in P^{\prime}$ denote $v(p)$ the direction of the bisector of $A(p)$, and $\mu(p)$ the unit direction of $x-p$. They give two maps $v: P^{\prime} \rightarrow S^{1}$ and $\mu$ : $P^{\prime} \rightarrow S^{1}$. The map $v$ is homotopic to the Gauss map of $P^{\prime}$, so $\operatorname{deg} v=1$. The map $\mu$ is homotopic to a constant map (the homotopy is obtained by moving $x$ to infinity without crossing $P^{\prime}$ ), so $\operatorname{deg} \mu=0$. Thus $v$ and $\mu$ should coincide on some $p$. The other (more elementary) way to prove the coincidence is to note that the rotation of the vector $v(p)$ when $p$ moves along $p^{\prime}$ is $2 \pi$, and the rotation of $\mu(p)$ is zero.

It is easy to see that for such $p$ the point $x$ lies inside $A(p)$, and the angular region $A(p)+x-p \subset$ int $A(p)$ gives what we need.

\section{Proof of Theorem 1}

Denote $\gamma=\min \{\operatorname{aco} K$, aco $L\}$. Consider the set $M=K+L$. Later we prove that it is simply connected (has no holes, i.e. bounded connected components of the complement $\mathbb{R}^{2} \backslash M$ ), but now we assume that the holes may exist and denote its outer piece of boundary by $B$ (the boundary of the component of $\infty$ of $\mathbb{R}^{2} \backslash M$ ). We are going to show that aco $B \geq \gamma$. 
Fig. 5 Points $p=a+b$, $q=c+d$, and the polyline $S=[c, a]_{\partial K}+{ }_{s}[d, b]_{\partial L}$

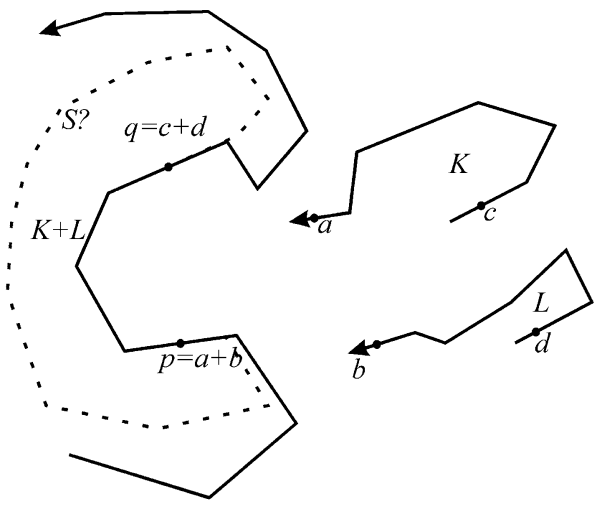

Suppose that for some two points $p, q \in B$ we have $\operatorname{rot}[p, q]_{B}=\delta<\gamma$ (the notation is the same as in the proof of Theorem 2). We may assume that $p$ and $q$ lie inside the edges of $B$. In this case from the definition of the Minkowski sum we deduce that

$$
p=a+b, \quad a \in K, b \in L, \quad q=c+d, \quad c \in K, d \in L .
$$

One of the points $a$ and $b$ (let it be $a$ ) lies on an edge (since $p$ lies on an edge) of $\partial K$ with outer normal $v$. The other point $b$ either lies on an edge of $L$ with the same normal, or is a vertex of $L$.

In the latter case the inner product $(\nu, x)$ on some small enough neighborhood of $b$ in $L$ attains its maximum exactly at $b$, in this case we can insert a "virtual edge" to $L$ of length zero at point $b$, so that it has normal $v$. This virtual edge does not affect the rotation of any subpolyline of $\partial L$. In the sequel we assume that $a, b, c, d$ lie on (possibly virtual) edges, the edges of $a, b, p$ have the same direction, and the edges of $c, d, q$ have the same direction.

Note that if aco $\partial K>-\pi$, then for any subpolyline $[x, y]_{\partial K}$ its rotation is less than $3 \pi$, otherwise the subpolyline $[y, x]_{\partial K}$ would have the rotation $\leq-\pi$. Now we see that $\operatorname{rot}[a, c]_{\partial K}=\delta+2 \pi N, N \in \mathbb{Z}$. From the angular convexity condition and the above note we deduce that $\operatorname{rot}[a, c]_{\partial K}=\delta+2 \pi$. So we have

$$
\operatorname{rot}[c, a]_{\partial K}=\operatorname{rot}[d, b]_{\partial L}=-\delta \text {. }
$$

Now consider the sorted sum $S=[c, a]_{\partial K}+{ }_{s}[d, b]_{\partial L}$. By Lemma 3 and Lemma 4 we have $\operatorname{rot} S=-\delta$, aco $S \geq \gamma>-\pi$. Lemma 1 shows that $S \subseteq M$. The situation is illustrated in Fig. 5.

Note that $M=\operatorname{clint} M$, int $M$ is connected, so we may put $S$ to general position so that it remains in $M$ and the only points of $S \cap \partial M$ are $p$ and $q$, we assume that the rotation of $S$ under this perturbation changes by arbitrarily small value, and aco $S$ remains $>-\pi$.

Then we eliminate loops on $S$ by Lemma 6 and obtain a simple polyline $S_{0} \subseteq M$ with rot $S_{0} \leq-\delta$. From Lemma 1 we know that $S_{0}$ goes from $q$ to $p$. Moreover, by the construction of the sorted sum its edge directions at $p$ and $q$ coincide (up to some small change on passing to general position) with the edge directions of $[p, q]_{B}$ 
(edges may be virtual). So the closed polyline $C=S_{0} \circ[p, q]_{B}$ has rotation number $\leq 0$ and does not have self-intersection.

Hence $\operatorname{rot} C=-2 \pi$, and the region that it bounds is to the right of $C$. But $C \subset M$, and along the polyline $[p, q]_{B}$, the set $M$ is to the left of $[p, q]_{B}$ ( $B$ is oriented anti-clockwise), which is a contradiction. Thus we have proved that aco $B \geq \gamma$.

Now we are going to prove that $M$ actually has no holes. Assume the contrary: a point $x \in \mathbb{R}^{2} \backslash M$ is not connected to $\infty$ in the complement of $M$. Let us perturb the vertices of $K$ and $L$ by values $<\delta$. If $\delta$ is chosen small enough then aco $K$, aco $L>$ $-\pi$ condition is kept, and $x$ remains outside $M$. Moreover, if $M$ does not have a hole containing $x$ after perturbation, then the complement $\mathbb{R}^{2} \backslash M$ contains by Theorem 2 an angular region $A$ with apex $x$ and bounded from below measure $\geq(\pi+\gamma) / 2$. Hence for small enough $\delta$ the region $A$ cannot be "blocked" by returning to the nonperturbed $K$ and $L$, we have a contradiction. Thus we have proved that for small enough $(<\delta)$ perturbations of $K$ and $L$ their sum $M$ still has a hole, i.e. the property of having a hole is open.

We are going to consider the parameterized family of polygons

$$
M(t)=K+t L .
$$

Note that the edges of $M(t)$ are contained in the lines (we define lines by two points)

$$
\left\{\left(v_{1}+t w_{1}, v_{2}+t w_{1}\right),\left(v_{1}+t w_{1}, v_{1}+t w_{2}\right)\right\},
$$

where $v_{1}, v_{2} \in K$ and $w_{1}, w_{2} \in L$ are vertices. By perturbing $K$ and $L$ we may require that all such lines (there is a finite number of them) have different directions. We also require that when $t$ changes in the range $(0,+\infty)$, no 4 of the lines meet at a single point, this can be achieved by perturbation.

If the parameter $t$ is close enough to zero, then the polygon $M(t)$ has the following structure: near an edge of $K$ it has a "long" edge, and near a vertex of $K$ it may be complicated. But since $K$ is an angular region $K \cap U(v)$ (possibly concave) in some neighborhood of a vertex $v \in K$, then $M(t)$ in $U(v)$ (and for small enough $t$ ) is a union of a family of translates of $K \cap U(v)$ that is starshaped. Hence, for small enough $t$, locally $M(t)$ has the same topology as $K$, and cannot have holes in global.

Now consider

$$
t_{0}=\inf \{t: K+t L \text { has a hole }\},
$$

it is already proved that $t_{0}>0$, assume $t_{0}<+\infty$. Since the "has hole" property is open, $M\left(t_{0}\right)$ has no holes. Consider what could happen if we increase $t$ by a small value $<\delta$. From the general position assumption $M(t)$ may be changed by adding some extra edge instead of a vertex, but this cannot give a hole. Hence $t_{0}=+\infty$ and the proof is complete.

\section{References}

1. Agarwal, P.K., Flato, E., Halperin, D.: Polygon decomposition for efficient construction of Minkowski sums. In: Lecture Notes in Computer Science, vol. 1897, pp. 20-31. Springer, Berlin (2000) 
2. Bennell, J.A., Dowsland, K.A., Dowsland, W.B.: The irregular cutting-stock problem-a new procedure for deriving the no-fit polygon. Comput. Oper. Res. 28(3), 271-287 (2001)

3. Burkea, E.K., Helliera, R.S.R., Kendalla, G., Whitwell, G.: Complete and robust no-fit polygon generation for the irregular stock cutting problem. Eur. J. Oper. Res. 179(1), 27-49 (2007)

4. Ivanov, G.E.: Weak convexity in the senses of Vial and Efimov-Stechkin. Izv. Math. 69(6), 1113-1135 (2005)

5. Pansu, P.: "Quasiconvex" domains in $\mathbb{R}^{n}$. In: Gromov, M. (ed.) Metric Structures for Riemannian and Non-Riemannian Spaces, pp. 393-400. Birkhäuser, Boston (2001). Appendix A 